\title{
United Parcel Service's IT Infrastructure: A Case Analysis
}

Russell Baker, Jacksonville University

Brian Wm. Dudley, Jacksonville University

Sean Holt, Jacksonville University

Chris Stockton, Jacksonville University

Vanja Vukota, Jacksonville University

\begin{abstract}
This analysis of the information technology infrastructure at United Parcel Service (UPS) begins with a general overview of the company's information technology (IT) environment to establish awareness of the size and complexity of this corporation. It includes a macro-level breakdown of the technology infrastructure of UPS ranging from the data centers to the PCs currently in operation. The study also more closely analyzes the software and database structure of UPS along with an analysis of the company's E-commerce activities. It includes an interview with Tom Creech, the North Florida District E-commerce manager at UPS. Finally, research was conducted to evaluate the emerging technologies that UPS is implementing and employing to support the business strategy and maintain its competitive edge.
\end{abstract}

\section{OVERVIEW}

nfrastructure, data and proprietary e-commerce software elements are the foundation of UPS's technology success. These tools work together to support its e-commerce strategy, which in turn supports the company's business strategy. The company's ongoing investment and research in emerging technologies gives UPS its competitive advantage according to Tom Creech, North Florida District E-commerce Manager. "UPS is always interested in current and emerging technologies that can assist our business and improve our efficiency" (Creech, 2004). Several awards have been presented to UPS for its application and innovativeness in e-commerce technology. InternetWeek's lists UPS in its 2004 top 100 companies. Infoworld ranked UPS seventh in its annual Infoworld 100, which represents the organizations with the greatest IT achievements (www.ups.com). UPS was analyzed for its effectiveness in using the information technology systems to achieve tangible business benefits. An analysis of the infrastructure, data and proprietary e-commerce tools is followed by a discussion of emerging technologies that UPS is integrating in to its information technology strategy.

\section{MACRO IT INFRASTRUCTURE}

The general network infrastructure of UPS, shown in appendix Figure 1, includes two data centers: the Ramap Ridge data center in Mahwah, New Jersey and the Windward data center in Atlanta, Georgia. The company uses 14 mainframes, 2,820 Mid-range computers, 120,000 PCs, 6,100 servers, and 80,000 wireless handheld Data Information Acquisition Device (DIADs); while its global telecommunications network has 2,445 network sites. The UPS website helps the company achieve many of its goals, both as a communication medium to individual customers and business clients and as a business portal to support e-commerce information exchange. The website receives an average of 115 million hits per business day and online tracking requests average 9.1 million inquiries per business day (www.ups.com). The Internet allows UPS to serve its customers in a more effective and efficient manner.

UPS rolled out its self-developed handheld Delivery Information Acquisition Device (DIAD) in 1990. The DIAD is a wireless handheld device that UPS drivers use to capture customer signatures, scan bar code tracking information, and transmit delivery-status information in real time directly to UPS's global network using a digital 
cellular connection (McDougall, 2004). The DIAD is able to process customer packages, time card reporting, and conduct time and motion studies to help improve the efficiency of the company (Ulfelder, 2002). These devices can transmit date to the UPS Mahwah data center three different ways: via an internal packet radio, a cellular modem in the truck and an internal acoustical coupler as used in an ordinary telephone line. The DIAD has triple communications redundancy that ensures real-time tracking in every environment and functions in conjunction with the company's network to capture and process data (Rosencrance, 2001).

Data captured by these handheld devices are transmitted to one of the two data centers in Mahwah or Atlanta. The network connecting UPS data centers is quite complex and extensive to be able to exchange data. It includes private links between all UPS facilities, internet interfaces, local area networks (LANS), and customer connections ranging from dial-up to dedicated lease lines within the overall network plan (Franklin, 2003). Businesses can be directly connected to the UPS global network enabling automated fulfillment and shipping processes directly linked between the business's computer system and the Mahwah data center (Rosencrance, 2001). The scope of the UPS IT infrastructure to capture, store, and retrieve data allows the company to maintain its competitive edge in the shipping industry.

\section{DATABASES}

According to UPS, the company operates the largest DB2 relational database in the world (www.ups.com). DB2 is an open source database developed by IBM. The Ramap Ridge data center in Mahwah NJ is used for essentially all of computing needs for UPS while Atlanta's Windward data center covers all of the shipping needs. (www.ups.com). The centers are self-sufficient and designed to provide redundancy for each other. The 18.41 terabytes database is sufficient to manage all information involved in tracking the packages and documents UPS delivers daily, plus all the other functions needed by UPS (www.ups.com). The databases are high-speed state-ofthe- industry systems, capable of reading 0.387 terabytes per hour (www.ups.com).

While exact information on how UPS configures its DB2database is proprietary, a typical configuration would utilize DB2's ability to enable both direct real-time access to data and batch processing. Typically comprised of a mainframe computer hosting databases and midrange servers hosting sub-databases that act as a communication tool between users and data stored on the mainframes, this setup ensures data security preventing unauthorized parties from accessing the main database. Another characteristic of DB2 is its ability to support nested tables, where data is arranged in rows and columns as in a spreadsheet. The ability to support nested tables allows DB2 to minimize indexing and maximize formatted efficiency (Bloor, 2004). DB2's DataJoiner allows joining data from different sources, which enhances sharing and manipulation of data by customers and UPS (Bloor, 2004). The information stored in the DB2 database together with information from other corporate functions is stored in the data warehouse, which has capacity of 7,618 terabytes, one of the largest databases in the world (www.ups.com). The amount of data that may be retrieved and processed on a daily basis from the data warehouse is up to 30,000 terabytes (www.ups.com).

UPS uses this computing power connected with UPS developed software to offer a variety of supply chain management services to other companies. UPS developed Quantum View software is a web-enabled application that allows for multiple users to access relevant information of the packages (www.ups.com). This application can also provide hourly updates about package information as well as track packages sent by multiple vendors.

Additionally, Quantum View instantaneously provides complete billing information to business customers. DaimlerChrysler is one of the UPS success stories involving the supply chain management team utilizing the UPS database and its Quantum View software. The UPS team was able to reengineer Daimler Chrysler spare parts delivery system, which provided significant performance improvement. Daimler Chrysler now uses UPS to ship and track its parts from its national distribution centers via UPS's consolidation centers that sort parts by dealership and then ship it to designated dealerships (www.ups.com).

Costa Rica based Ikor is a similar success story. The company was shipping almost 3,000 units per week and was in dire need for a logistics upgrade to improve delivery performance. Ikor partnered with UPS by using its 
supply chain management group and the UPS Trade Direct Cross Border software that consolidates orders, routs them for transport and expedites customer clearance through customs, saving Ikor and their customers time and money (Malykhina, 2004). Paul Lesniak, chairman and CEO of Ikor said, "Having a single point of contact for all its logistic needs made a world of difference to Ikor" (Malykhina, 2004). This outsourcing has enabled Ikor to focus on its core business and not on logistics, leaving that to UPS and its technological supply chain team.

\section{UPS ONLINE TOOLS}

In April 6 1999, UPS deployed Online Tools. Online Tools allows customers and systems integrators to seamlessly link with UPS through their business's intranets and Internet websites. These systems would allow the customer to improve customer service by lowering costs and keeping its own customers focused on their ecommerce website. Components of Online Tools include:

Enhanced Tracking - This powerful tool that enables shipment tracking from the shipper's E-commerce site using a purchase order or other reference number, thereby improving customer service and enhancing UPS's competitive stance. The UPS customer is able to ascertain where its package is in the shipment process, thereby providing better customer service, and thus supporting the strategic initiative of UPS (UPS E-commerce Site).

UPS Signature Tracking - This tool enables customers to track packages and obtain proof of delivery when the shipment is complete. Customers receive the digital signature of the individual recipient along with delivery address, amount collected (where appropriate), and the delivery reference. The system allows UPS customers to instantly obtain an official proof of delivery when needed. This system can also improve cash flows due to shorter billing cycles while allowing better customer service and information to clients of UPS's business customers. Proof of delivery is known instantly, and therefore payment can be received sooner (UPS E-commerce Site).

UPS Online Tools - Online Tools, available in both HTML and XML formats, are easy to integrate and downloadable from UPS's website. The XML standard facilitates shipping functionality integration into UPS's customers systems, thereby providing enhanced flexibility and mass customization capabilities (UPS E-commerce Site).

\section{UPS SHIPPING SOLUTIONS}

UPS's core business is package delivery the company has developed additional online tools customers use to access all information on their packages in real-time. Such tools compliment and are additions to the UPS Online Tools package discussed earlier.

UPS WorldShip - WorldShip is proprietary software that allows shippers to manage all shipping activities from their own PC. From creating a label, generating export information, and validating address information, WorldShip can get the customer to "manage their journey from origin to destination- and back again if necessary!" (UPS Ecommerce Site).

UPS CampusShip - CampusShip is a secure multiple-user Web-based shipping system that enables shipping packages from any Internet accessible computer in addition to performing other UPS shipment-related tasks. This web-based tool that can be used anywhere in the organization that requires minimal training and maintenance (UPS E-commerce Site).

\section{EMERGING TECHNOLOGIES}

The UPS e-commerce model is propelled by is continued investment in operational efficiencies. Gains in efficiencies can be directly attributed to the development and deployment of the latest technology. UPS has a robust technology research and development department that helps support its strategic operating goals. Several emerging technologies are shaping the operational future of UPS. These are discussed here. 


\section{DIAD IV}

UPS is planning a quantum leap in the DIAD capability, specifically installing GPS satellite tracking systems in the DIAD IV to monitor and analyze the routes to reduce costs of transporting the packages and to provide better customer service. If a customer calls with a last-minute change of address, the package could be rerouted in transit using a geospatial application that would help the driver find the most efficient route to the new location (Gruman, 2004).

The Delivery Information Acquisition Device Generation 4 (DIAD IV) represents a significant step forward for UPS Technologies. The DIAD IV builds on the success of its predecessors, but it has whole new platform and design. The development of the DIAD IV will cost UPS \$22 million and will be produced by Symbol Technologies Inc. It is expected that over 70,000 will be deployed to delivery drivers worldwide (Brewin, 2003). The DIAD IV is built on a lightweight platform that has a large color screen, alphanumeric keypad and softkeys. The unit is smaller than the Motorola built DIAD III, carries more features and can operate in more technology environments. The new DIAD IV runs Microsoft.Net on a Windows CE operating system and has 128 megabytes of memory. The system's connectivity options include the familiar General Packet Radio Service and dial-up access that were used in past models as well as Code Division Multiple Access (CDMA, cellular) radio for metropolitan communications and WLAN using native $802.11 \mathrm{~b}$ for transmitting in UPS distribution centers. The new model will also include short-range Bluetooth and Infrared communications for interfacing with customer systems (Bednarz, 2004). The DIAD IV is reportedly the first handheld computer to communicate on three network protocols: Bluetooth, cellular-radio, and infrared, outpacing rival FedEx, and will ensure that the device will be able to communicate wherever it is (UPS press release, 2003). The DIAD IV will also include Global Positioning Systems (GPS) functionality. The GPS feature will allow the device to suggest routes and will track the driver's movements. Drivers also receive visual and audible alerts for special pickups and traffic reports, record their time and attendance and can load special parking instructions, security procedures and customer contact information that can be reviewed by other drivers before visiting a particular customer location (Interview with Tom Creech, 2004). Bar code scanners and the signature capture process are used by the older and newer models, but new features like receipt printers and credit card readers are expected to be in the roll-out only in the DIAD IV (UPS press release, 2003). The new model also incorporates data security and driver safety features as well. It is expected that the new DIAD IV will yield gains in information and billing accuracies, pick-up and delivery timeliness, and route planning thus reducing fuel costs and improving customer service and driver courtesy measures.

\section{Radio Frequency Identification (RFID)}

The use of radio frequency identification (RFID) at UPS began in 1985. Although the technology is not new to the firm, its use is just now beginning to be fully implemented. RFID, which uses special frequency scanners to "read" miniature radio transmitters that are imbedded in objects, is the latest in inventory tracking and supply chain management methods on the market. UPS is working with Alien Technology Corp. and Symbol Technologies Inc. to develop ways to use RFID in its package shipping business (Malykhina, 2004). The technology is widely expected to replace the bar code technologies that nearly all of today's package shipping firms use.

RFID systems in use today use a mixture of passive tags energized by a local reader/scanner and active tags that contain their own battery that operating on Class $0(125-\mathrm{KHz})$ or Class $1(13.56-\mathrm{MHz})$ frequencies. Older RF tags have substantial ROM (read only memory) memory limitations, but new tags can hold 30 times more information than older tags, which is enough ROM memory to hold a package's complete shipping information (Brewin, 2002). UPS is experimenting with these technologies at its Atlanta distribution centers according to David Barnes, Vice President of IS at UPS in Atlanta (Bednarz, 2004). The firm envisions a day when each package can be tracked using RFID, but the firm has good reason to hold off on these plans. Industry has not fully adopted this technology given that RFID labels are much more costly than a paper bar code labels; they can range from $\$ .40$ to over a dollar per tag (Roberti, 2004). Currently UPS has utilizes tags for its pallets. These tags are more durable, usually three years, than bar codes and do not require line-of-sight as bar code reading. Another reason that RFID is still in pilot stages at UPS and many other firms is that there are no agreed-upon standards or development roadmaps. As a leading user of RFID, UPS has joined the Strategic Enterprise Fund, an industry standards group 
working to develop best practices and standards for use of RF technologies. Frequencies used in RF over air protocol range from low frequency, which is used for long distances to ultra high frequency (UHF Gen 2 as it is called), which is still under development (Brewin, 2002).

Additionally, the current RFID tags cannot provide sequence nor exact location information which is two features that UPS needs to accurately queue and properly load its delivery trucks and airplanes. UPS does use pallets and cartons tagged with RFID labels. UPS has developed a way to track individual packages as they move through its Atlanta warehouse by recording which pallet it is on while tracking the pallet. RFID tags do register information much more quickly than bar codes because they can be polled simultaneously rather than sequentially. UPS is also piloting the use of RF tags at UPS truck corrals and fueling depots.

\section{WLAN And Bluetooth}

UPS is investing in its network at its corporate offices and 2000 plus distribution centers. The firm is using a wireless local area network (WLAN) based on native $802.11 \mathrm{~b}$ and Microsoft CE powered wireless devices. UPS is partnering with Symbol Technologies Inc., the same firm that is supplying the DIAD IV, to provide on site wireless solutions. The WLAN will require over 15,000 access points. It is estimated that the cost of the Wireless LAN project will exceed $\$ 100$ million with an expected payoff of 16 months (Mobileinfo.com, 2002). Wireless networking is more cost effective than traditional wired LANs especially given the amount of twisted pair cable it would take to network all of UPS's immense distribution centers. Wireless LANs also enable the use of wireless scanners and readers that are deployed on fork trucks, pallet jacks and in the hands of package handlers throughout the enterprise (Mobileinfo.com, 2002). UPS will start employing a new Bluetooth enabled cordless ring scanner that slips on to the hand of a package sorter. This innovation from Symbol Technologies, Inc. will replace the current handle held scanners, thus freeing up the sorters hands to move boxes, while continuing the scanning function (Mobileinfo.com, 2002).

\section{SUMMARY AND FINDINGS}

Three main elements make up UPS's e-commerce environment: infrastructure, data and proprietary ecommerce software. These elements, taken together produce a strong e-commerce system that supports the company's business strategies/mission. UPS is constantly investing in emerging technologies in order to improve its operations and meet its mission, to be the leading delivery service of packages and documents in the United States and throughout the world. UPS's commitment to utilize leading edge technology to improve service delivery, implementing improved and efficient business processes and capitalizing on business opportunities leads us to believe that UPS will be the dominant package and document delivery service for many years to come.

\section{REFERENCES}

1. Bednarz, Ann, (2004) [Electronic version]. Network World, Retrieved Nov 7, 2004 http://www.nwfusion.com/news/2004/092004upsrfid.html?page=1

2. $\quad$ Brewin, Bob, (2002) [Electronic version]. Computer World, Retrieved Nov 7, 2004 http://www.keepmedia.com/pubs/Computerworld/2002/12/16/305492

3. Brewin, Bob, (2003) [Electronic version]. Computer World, Retrieved Nov 7, 2004 http://www.computerworld.com/mobiletopics/mobile/

4. Creech, Tom. (2004) Personal Interview with the North Florida District E-commerce Manager, UPS, October 2004.

5. Franklin, Curtis, (2004) [Electronic version]. ComNews, Retrieved Oct 31, 2004 http://www.comnews.com/stories/articles/c0404cover.htm

6. Gruman, Galen, (2004) [Electronic version]. CIO Magazine, Retrieved Sept 12, 2004 http://www.cio.com/archive/060104/ups.html

7. Howard, Philip, [Electronic Version] http://www.bloor-research.com

8. Malykhina, Elena, (2004) [Electronic Version]. Information Week, Retrieved October 18, 2004 http://www.informationweek.com/showArticle.jhtml?articleID=49901762 
9. McDougall, Paul, (2004) [Electronic version]. Information Week, Sept 12, 2004

http://informationweek.com/shared/printableArticleSrc.jhtml

10. Roberti, Mark, (2004) [Electronic version]. RFID Journal, Retrieved Nov 7, 2004

http://www.rfidjournal.com/article/articleview/943/1/1

11. Rosencrance, Linda (2001) [Electronic version]. Computer World, Sept 12, 2004 http://www.computerworld.com/printthis/2001/0,4814,58696,00.html

12. Ulfelder, Steve, (2002) [Electronic version]. Computer World, Retrieved Sept 12, 2004 http://www.computerworld.com/printthis/2002/0,4814,74625,00.html

13. UPS E-commerce website, UPS corporate website, 2004 Retrieved Nov 7, 2004 http://www.ec.ups.com/ecommerce/solutions/c2.html

14. UPS press release, UPS corporate website, May 16, 2003 Retrieved Nov 7, 2004 http://www.pressroom.ups.com/

15. UPS press release, (2004) UPS corporate website, February 4, 2004. Retrieved Nov 4, 2004 http://www.pressroom.ups.com/

16. UPS press release, UPS corporate website, May 16, 2003 Retrieved Nov 7, 2004 http://www.pressroom.ups.com/

17. UPS Technology Facts. Retrieved Oct 10, 2004 Retrieved Nov 4, 2004 http://www.ups.com/content/us/en/about/facts/technology.html

18. UPS press release, (2003) UPS corporate website, 15 April 2003 Retrieved Nov 7, 2004 http://www.pressroom.ups.com/pressreleases/archives/archive/0,1363,4287,00.html

19. UPS Uses WiFi \& Bluetooth Together to Manage Packages at Shipping Hubs, Issue \#2002, (December 2002) Retrieved Nov 7, 2004 http://www.mobileinfo.com/News_2002/Issue47/UPS_WiFi_Bluetooth.htm 


\section{APPENDIX}

Figure 1 - UPS Regional Network

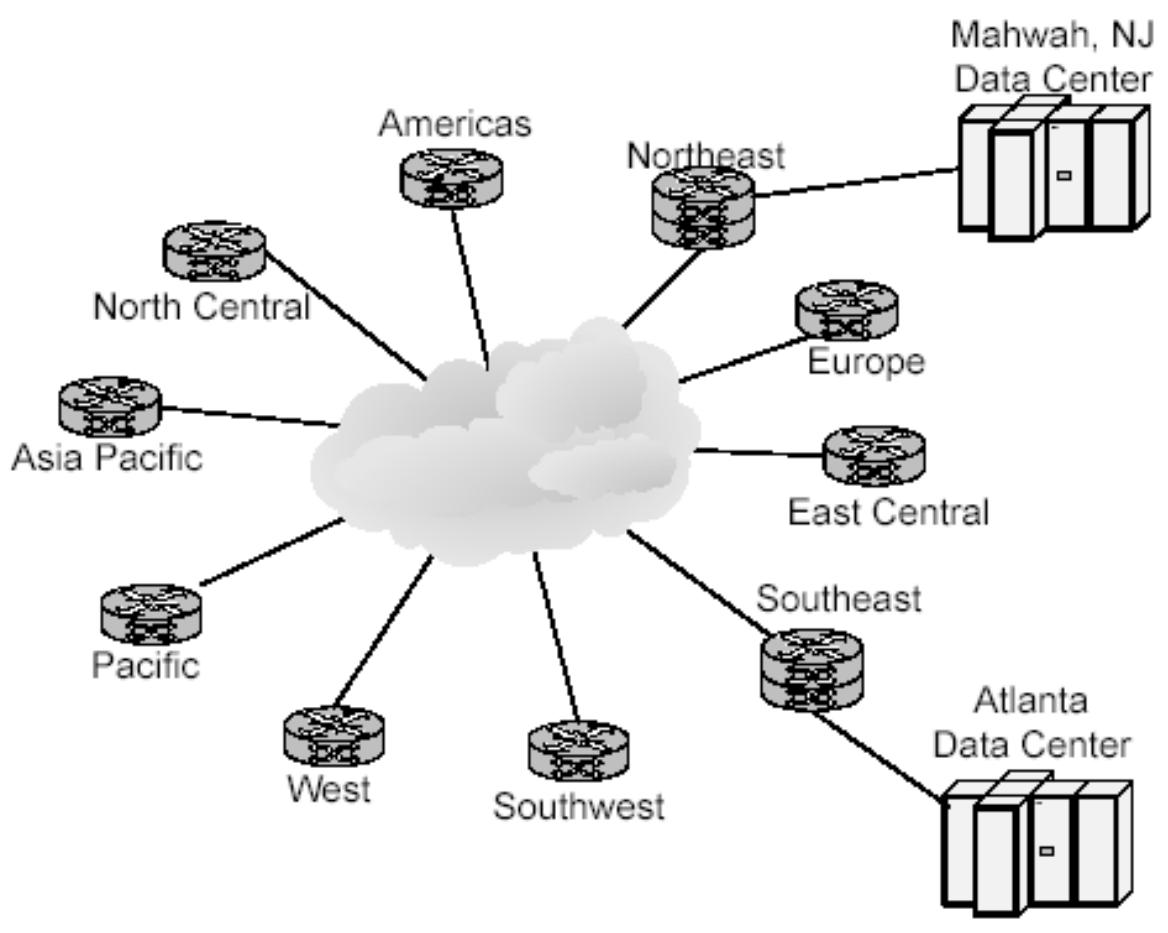

Figure 2 - UPS Site Network

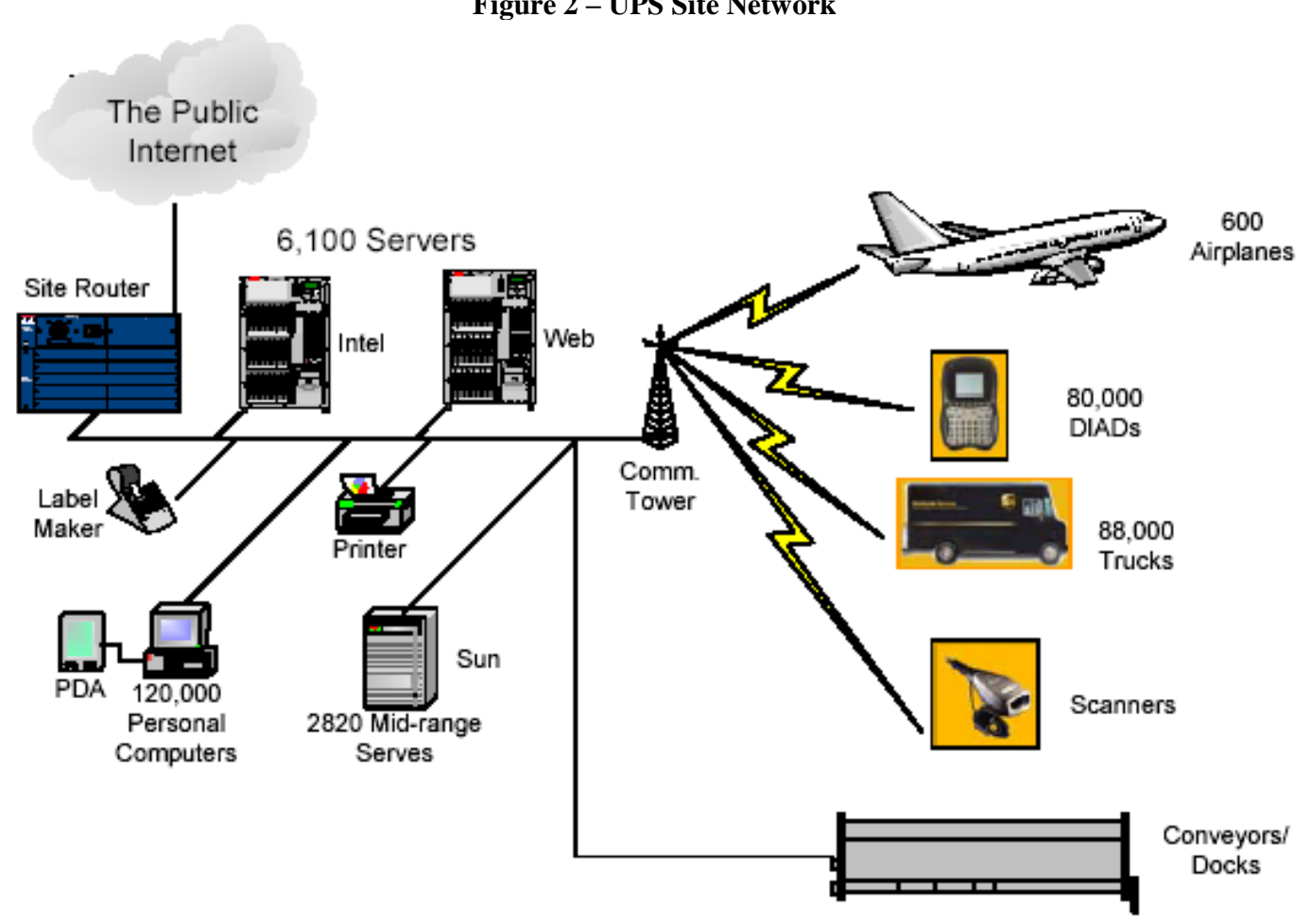


NOTES 